Supplemental Table 1: Cytosolic ribosomal proteins O: OSMO, U: 8M urea, C: CHAPS

\section{Eukaryotic translation initiation}

translation elongation factor eEF-2 translation initiation factor elF-4A.9 eukaryotic initiation factor $4 \mathrm{~A}-1$ eukaryotic initiation factor $4 \mathrm{~A}-2$ eukaryotic initiation factor $4 \mathrm{~A}-10$ eukaryotic initiation factor $4 \mathrm{~A}-15$ elongation factor 1-alpha elongation factor 2

elongation factor EF-2

\section{Ribosomal proteins}

40S ribosomal protein $\mathrm{S} 13$

40S ribosomal protein S14

$40 S$ ribosomal protein S4

$60 S$ ribosomal protein $\mathrm{L} 2$

40S ribosomal protein $\mathrm{S} 16$

$60 S$ ribosomal protein $\mathrm{L} 13$

$60 S$ ribosomal protein $\mathrm{L} 17$

$60 S$ ribosomal protein $\mathrm{L} 15$

$40 S$ ribosomal protein S8

$60 S$ ribosomal protein L6

$60 S$ ribosomal protein L9

60 S ribosomal protein $\mathrm{L} 27$

$40 S$ ribosomal protein-like

$60 S$ ribosomal protein $L 7 A$

$60 S$ ribosomal protein $L 1$

$60 S$ ribosomal protein $L 23 A(L 25)$

$60 S$ ribosomal protein $\mathrm{L} 3$

$40 \mathrm{~S}$ ribosomal protein $\mathrm{S} 5$

ribosomal protein L12

ribosomal protein $\mathrm{S} 19$

ribosomal protein L7

ribosomal protein $\mathrm{S} 2$

similar to Arabidopsis thaliana 605 ribos gi| 7340874 gi|7443459

gi|2119932

gi|1170503

gi|1170506

gi|1170511

gi|2500521

gi|1169476

gi|461998

gi|6056373

gi|1173198

gi|1173200

gi|1173256

gi|132849

gi|133808

gi|1350664

gi|1710519

gi|3122673

gi|3777552

gi|464621

gi|6174959

gi|730547

gi|7488177

gi|548774

gi|4887131

gi|585876

gi|132944

gi|6831665

gi|3986695

gi|445612

gi|445613

gi|7110459

\section{Fraction}

C

U

U

C

U

C

C

C

C

C

C

C

C

C

C

C

C

C

C

C

C

C

C

C

C

U

U

C

C

U

C 